\title{
PROBLEM-SOLVING ABILITY TESTED
}

\section{ICCA Communication \\ by Gorran Grottling}

It is well-known that chess computers are very good at solving problems, often finding the solution before a human being has had the time to become familiar with the problem. Even Grand Masters have had to bite the dust.

As far back as the era of Chess Challenger 10, its ability to solve mate-in2 problems was impressive. Nowadays, of course, the best machines outperform Chess Challenger 10 by factors of many hundreds.

We have tested 18 chess programs on 16 arbitrarily chosen problems by Samuel Loyd. We found the following ranking for the top fourteen:

$\begin{array}{lllr}\text { 1. Conchess 4 MHz } & 914 & \text { 8. Turbostar } & 115 \\ \text { 2. Conchess 2 MHz } & 458 & \text { 9. Mark V } & 99 \\ \text { 3. Matebadix Intelligent } & 438 & \text { 10. Constellation 2.0 MHz } & 98 \\ \text { 4. Super Constellation } & 223 & \text { 11. Mephisto Exclusive S } & 86 \\ \text { 5. Constellation } 3.6 \mathrm{MHz} & 177 & \text { 12. Prestige } & 39 \\ \text { 6. Elite A/S } 3 \mathrm{MHz} & 151 & \text { 13. Capablanca } & 30 \\ \text { 6. Elegance } 3.68 \mathrm{MHz} & 151 & \text { 14. Mephisto III Exclusive } & 23\end{array}$

The number of points behind the ranking has been arrived at simply: the fastest program to solve any problem was given 1,000 points. Competitors were given points inversely proportional to their solution times. The results above are averages over the 16 problems. The ranking scheme may be challenged - your proposals are invited.

The table compares speed only for those computers which indeed were capable of solving all problems set. (Four failed and, while eliminated in the table. above, stili have such solutions as they did find recorded below.) Evidently Conchess outdoes all its competitors in problem solving. This may be related to their programming team Ulf Rathsman and Johan Enroth having a penchant for chess problems.

A few words are in order on Matebadix Intelligent. Ilka Blom, from Finland, programmed it (see ICCA Journal, Vol. 7, No. 1, pp. 56-57) and it is now commercially available on diskette and tape for the Commodore 64 and other computers, with Apples having a slight time advantage. Using accelerator cards, speeding up the processors by a factor of 3.5, would promote Matebadix Intelligent to the top of our list since the Commodore 64 is no faster than around $1 \mathrm{MHz}$.

In conclusion, we may state that there are vast time differences in problemsolving ability. For the 14 computers 1isted, they do not affect the quality of solutions, since all of them are up to Samuel Loyd. The time differences are mainly ascribable to processor rates and programming tactics. A 'Which?' ranking would dissuade intending customers from buying any of the programs not 1 isted above and urge them to acquire programs by their stated ranking whenever problem-solving speed is of the essence. 
MATE-IN-2 PROBLEMS

$\underline{\text { Problem } 1}$

White: Kh1 Qa8 Ra5 Rh5 Bb3 Bg7 Ne4 b6 d7 e6 f3 g3;

Black: Kb4 Qg6 Rd3 Rf8 Bd8 Ne1 c2;

Solution: 1. Rf5

\section{Problem 2}

White: Kh7 Qa3 Re3 Rf2 Bg2 Bh2 Nd1 Nd2 c2 e5 g6;

Black: Kd4 Qc8 Rc1 Rf6 Bbl $\mathrm{Ng} 7 \mathrm{Ng} 8$ c4 d6 g5 h4;

Solution: 1. Rg3

\section{Problem 3}

Wh1te: Kf1 Qa4 Rb2 Rh1 Ba3 Bd3 Ng2 Nh3 e2 f3 g3 h4;

Black: Kc1 Qh6 Rd8 Rh8 Bb7 Bg7 Ne1 a7 b6 c7 f7 h7;

Solution: 1. Bf8

\section{MATE-IN-3 PROBLEMS}

Problem 4

White: Kh3 Qc2 Rb2 Rf8 Bal Bf3 Nd1 Nf5 c5 h4;

Black: Ke5 Qa2 Ra8 Rf4 Bb7 Bg3 Nc1 Nc6 d6 d5 e6;

Solution: 1. Qh2

\section{$\underline{\text { Problem } 5}$}

White: Kf1 Ra5 Rf6 Bb5 Bg7 Nb6 Ne4 b4 d2;

B1ack: Ke5 Rc8 Rh2 Bg3 Bg8 Na2 Nh1 a6 b7 c3 e6 f2 h4;

Solution: 1. $\mathrm{Ke} 2$

Problem 6

White: $\mathrm{Kh} 7 \mathrm{Rd} 7 \mathrm{Re} 2 \mathrm{Ba} 6 \mathrm{Bc7} \mathrm{Nb} 5$ a4 b3 g4;

Black: Kc5 Rh2 Ba8 Nf3 Nh1 a5 a7 b4 c6 g5 g7 h4;

Solution: 1. Bb7

\section{Problem 7}

White: $\mathrm{Kg} 8 \mathrm{Rb} 3 \mathrm{Re} 3 \mathrm{Bd} 4 \mathrm{Bf} 1 \mathrm{Nd1} \mathrm{Nf} 7$ c7 f2 $\mathrm{g} 3$;

Black: Kg4 Qc1 Rh6 Ba5 Nh5 Na4 Na7 c5 d2 d5 e4 g6 h3 h7;

Solution: 1. Rb6

\section{MATE-IN-4 PROBLEMS}

\section{$\underline{\text { Problem } 8}$}

White: $\mathrm{Ke} 1 \mathrm{Qd4} \mathrm{Bg} 3 \mathrm{Na6} \mathrm{Nd6}$ e5;

B1ack: Kc6 Ba2 Na8 b5 b6 d7 e6 f7 g6;

Solution: 1. Qg1 


\section{Problem 9}

White: Ka8 Qh8 Rg2 Bel Bf1 Nh4 a5 b6 f4 g5 h6;

B1ack: Kh3 Qc6 Na2 Nb1 a6 b7 d5 e4;

Solution: 1. Bxa6

Problem 10

White: Ka3 Rd4 Rh1 Bcl Bd1 Nc3 Nd3 b2 b6;

Black: Kal Qb1 Rd7 Rd8 a4 b7 c6 d5;

Solution: 1. Rdh4

Problem 11

White: Kh6 Ral Rd8 Bg8 Na5 Nc4 c2 d2 e6 f3 f4;

Black: Kc5 Bb8 Nc7 a7 a6 e7;

Solution: 1. Rc8

\section{MATE-IN-5 PROBLEMS}

\section{Problem 12}

White: Kcl Ra2 Rf1;

Black: Kh1 Bh1 a5 h2;

Solution: 1. Raf 2

Problem 13

White: $\mathrm{Ke} 7 \mathrm{Rh} 3 \mathrm{Bd} 4 \mathrm{~g} 4$;

Black: $\mathrm{Kg} 7 \mathrm{Rf} 6 \mathrm{Bg} 8 \mathrm{f7}$;

Solution: 1. Rh6

Problem 14

White: $\operatorname{Ke1} \mathrm{Bh} 6$ c2 e2;

Black: $\mathrm{Kg} 1 \mathrm{Qh} 1 \mathrm{Rg} 3 \mathrm{Ba} 8 \mathrm{c} 3 \mathrm{~g} 2 \mathrm{~h} 2 \mathrm{~h} 5$;

Solution: 1. Bcl

\section{Problem 15}

White: Kal Rf8 Nd3 Bb5 Bc5 d6;

Black: $\mathrm{Kd} 5 \mathrm{Rg} 7$;

Solution: 1. $\operatorname{Re} 8$

\section{MATE-IN-6 PROBLEM}

White: Kf2 Ne2 Nf5;

Black: Kh1 Na6 Nb6 c5 e7 h7;

Solution: Nh6

[Problem positions are presented algebraically for programmers' convenience; the Swedish equivalent of this contribution may be found in PLY, 1985, No. 2.] 


\begin{tabular}{|c|c|c|c|c|c|c|c|c|c|c|c|c|c|c|c|c|}
\hline Problem & $\underline{1}$ & $\underline{2}$ & $\underline{3}$ & $\underline{4}$ & $\underline{5}$ & $\underline{6}$ & $\underline{7}$ & $\underline{8}$ & $\underline{9}$ & 10 & 11 & 12 & 13 & $\underline{14}$ & 15 & $\underline{16}$ \\
\hline Conchess $4 \mathrm{MHz}$ & 2 & 2 & 2 & 16 & 14 & 5 & 20 & 112 & 66 & 498 & 20 & 602 & 786 & 56 & 3119 & 921 \\
\hline Conchess $2 \mathrm{MHz}$ & 4 & 4 & 3 & 33 & 27 & 11 & 41 & 227 & 133 & 1014 & 42 & 1222 & 1594 & 114 & 6240 & 1980 \\
\hline Matebadix Intell. & 4 & 7 & 6 & 54 & 32 & 37 & 78 & 641 & 783 & 756 & 445 & 810 & 322 & 72 & 685 & 3347 \\
\hline Super Constellation & 5 & 7 & 4 & 93 & 70 & 22 & 79 & 960 & 858 & 2550 & 499 & 1489 & 1730 & 111 & 10000 & 7710 \\
\hline Constellation 3.6 & 7 & 8 & 4 & 142 & 99 & 31 & 103 & 1340 & 1453 & 4004 & 993 & 2422 & 3047 & 155 & 13558 & 6192 \\
\hline Elite $\mathrm{A} / \mathrm{S} 3 \mathrm{MHZ}$ & 4 & 5 & 7 & 291 & 147 & 94 & 150 & 3423 & 3196 & 4800 & 2649 & 1951 & 10080 & 238 & 69900 & 6240 \\
\hline Elegance $3.68 \mathrm{MHz}$ & 4 & 5 & 7 & 251 & 119 & 87 & 144 & 2475 & 2465 & 3202 & 1947 & 2947 & 2654 & 323 & 151440 & 8938 \\
\hline Turbostar & 15 & 16 & 10 & 292 & 92 & 73 & 178 & 1330 & 10705 & 6162 & 498 & 4555 & 5275 & 650 & 1502 & 17985 \\
\hline Mark V & 11 & 22 & 20 & 156 & 134 & 101 & 235 & 2425 & 3801 & 4499 & 2694 & 2495 & 8782 & 254 & 4330 & 28243 \\
\hline Constellation 2.0 & 12 & 14 & 8 & 230 & 178 & 56 & 185 & 2528 & 2617 & 7209 & 1848 & 4342 & 5486 & 280 & 24402 & 11148 \\
\hline Mephisto Excl. S & 19 & 29 & 26 & 250 & 202 & 279 & 379 & 2490 & 998 & 2101 & 1172 & 5035 & 3453 & 264 & 5765 & 81180 \\
\hline Prestige & 24 & 84 & 29 & 4855 & 1726 & 317 & 2639 & 13865 & 53230 & 9440 & 13096 & 14550 & 17361 & 223 & 180734 & 25460 \\
\hline Capablanca & 35 & 30 & 30 & 720 & 448 & 176 & 474 & 4781 & 4231 & 35964 & 4318 & 11112 & 21792 & 1949 & 356422 & 79267 \\
\hline Mephisto III Excl. & 76 & 109 & 100 & 958 & 817 & 1103 & 890 & 9720 & 3900 & 8017 & 4642 & 18300 & 15130 & 928 & 29892 & 182220 \\
\hline Sensory 9 B & 37 & - & - & - & 120 & - & 110 & - & - & 2220 & 730 & 1990 & 11640 & 310 & 3000 & 31920 \\
\hline Chess 2001 & 28 & 35 & 22 & 300 & 295 & 308 & 500 & 4725 & 10408 & 15437 & 6380 & - & - & - & - & - \\
\hline Morphy & 60 & 87 & 35 & 2697 & 349 & 431 & 655 & - & - & - & - & - & - & 1505 & - & - \\
\hline Boris 2.5 & 138 & 177 & 130 & 5472 & 1302 & 1014 & 2303 & - & - & - & - & & & - & & \\
\hline
\end{tabular}

The solution times have been given in seconds, counting to the instant when the computer declared its solution to be final. Dashes refer either to no solution being found or to 'solutions' involving too many moves. 